\title{
Using Hammock Graphs to Structure Programs
}

\author{
Fubo Zhang and Erik H. D'Hollander, Member, IEEE
}

\begin{abstract}
Advanced computer architectures rely mainly on compiler optimizations for parallelization, vectorization, and pipelining. Efficient code generation is based on a control dependence analysis to find the basic blocks and to determine the regions of control. However, unstructured branch statements, such as jumps and goto's, render the control flow analysis difficult, time-consuming, and result in poor code generation. Branches are part of many programming languages and occur in legacy and maintenance code as well as in assembler, intermediate languages, and byte code. A simple and effective technique is presented to convert unstructured branches into hammock graph control structures. Using three basic transformations, an equivalent program is obtained in which all control statements have a well-defined scope. In the interest of predication and branch prediction, the number of control variables has been minimized, thereby allowing a limited code replication. The correctness of the transformations has been proven using an axiomatic proof rule system. With respect to previous work, the algorithm is simpler and the branch conditions are less complex, making the program more readable and the code generation more efficient. Additionally, hammock graphs define single entry single exit regions and therefore allow localized optimizations. The restructuring method has been implemented into the parallelizing compiler FPT and allows to extract parallelism in unstructured programs. The use of hammock graph transformations in other application areas such as vectorization, decompilation, and assembly program restructuring is also demonstrated.
\end{abstract}

Index Terms-Program transformation, structured programming, compilers, optimization, parallel processing, software/program verification, correctness proofs.

\section{INTRODUCTION}

A structured program is a block-oriented program in which each block of statements has a single entry, a single exit, and the blocks are properly nested. Using blockoriented control constructs, such as while and if-then-else statements, most imperative languages enforce a structured style. However, branches such as jumps and goto's, interacting with block-oriented statements, make these statements unstructured, because there is no single locus of control.

The work presented here describes a method to convert programs with unstructured branches into structured form. The motivation is that structuring is necessary for a compiler to perform optimizations such as parallelization and vectorization. To this end, we present a new restructuring algorithm which limits code expansion and maintains a simple control structure. The algorithm eliminates all branches, maintains the basic block lengths, preserves structured loops and if-tests, and introduces only a few logical variables. The new approach is based on the creation of nested hammock graphs. These single-entry, single-exit graphs create well-defined nested control regions which can be separately optimized and parallelized. The method has been implemented in a parallelizing compiler and was able to generate a significant number of extra parallel loops.

- F. Zhang is with Platform Computing Inc., 3760 14th Ave., Markham, Ontario L3R 3T7, Canada. E-mail: zfb@platform.com.

- E.H. D'Hollander is with the Department of Electronics and Information Systems, Ghent University, St.-Pietersnieuwstraat 41, B-9000 Ghent, Belgium. E-mail: dhollander@elis.ugent.be.

Manuscript received 26 Aug. 2003; revised 23 Jan. 2004; accepted 29 Jan. 2004.

Recommended for acceptance by J. Knight

For information on obtaining reprints of this article, please send e-mail to: tse@computer.org, and reference IEEECS Log Number TSE-0121-0803.
Furthermore, examples illustrate that the resulting code is generally more readable.

This research grew out of the need to parallelize and vectorize unstructured programs. The resulting restructuring method is simple, implementable in a compiler, provably correct, and generates a less complex control flow. A major benefit is that hammock graph restructuring builds a hierarchy of structured code regions. This enlarges the granularity and makes global code optimization possible, such as loop transformations, data locality enhancements, and other techniques used to find parallelism [14], [21], [38]. It is well known that unstructured code such as irreducible loops, i.e., loops with more than one entry point [16], may block optimization because there is no information on the control flow [15]. Existing front-end compiler methods are directed towards loop normalization [3] and goto elimination [13]. Back-end methods repair irreducible loops by node splitting (replication) and DJ-graphs [19], [32]. The present method is a front-end approach which repairs unstructured regions by creating hammock graph structures in the control flow graph.

Hammock graph restructuring operates on the control flow graph of the source code and eliminates all branches while minimizing the number of new control variables and associated control expressions. As a consequence, the basic blocks keep the same length as in the original program and the branching conditions maintain the same complexity. The conversion of the program is based on three elementary transformations, namely, the backward copy, forward copy, and cut operations. The transformations reorganize the program into a set of nested hammock graphs, each having a single entry and a single exit. As an example, consider the program in Fig. 1. The loop created by goto 100 has two entries and is therefore irreducible. After a backward copy Fig. $1 \mathrm{~b}$ and a simple if-conversion Fig. 1c, the resulting 


\begin{tabular}{|l|l|l}
\hline if (x) goto 200 & if (x) goto 200 & if (.not. $x)$ then \\
$100 \mathrm{~S} 1$ & $100 \mathrm{~S} 1$ & S1 \\
$200 \mathrm{~S} 2$ & $200 \mathrm{~S} 2$ & endif \\
if (y) goto 100 & do while(y) & S2 \\
& S1 & do while(y) \\
& S2 & S1 \\
& enddo & S2 \\
& & enddo
\end{tabular}

(a)

(b)

(c)

Fig. 1. (a) Irreducible loop with two statements S1, S2, (b) after backward copy, (c) after single branch to if-conversion.

program contains two hammock graphs. In contrast to the original program Fig. 1a, the structured program enables optimizations which are otherwise impossible due to lacking control flow information. For example, the while loop may be converted into a parallel loop subject to a dependence analysis [38]. Further examples of optimization are given in Section 5 .

The correctness of the three transformations has been proven using an axiomatic proof rule system. The remainder of this text is organized as follows: In Section 2, definitions and a basic corollary are given. The methods for converting branches into block structured control statements are developed in Section 3. Here, program transformations for single branches, multiple interacting branches, and branches interacting with structured control statements are developed. The correctness of the program transformations is formally proven in Section 4 using axiomatic proof rules for branches. A number of experimental results in the area of parallel processing are reported in Section 5. In Section 6, the related work is described and compared with the proposed approach. Concluding remarks are given in Section 7.

\section{Definitions}

A program consists of sequential statements and control statements. During the execution, a sequential statement is followed by the next statement in the program whereas the statement following a control statement is selected by a conditional jump. The statement ordering during the execution of the program is described by the control flow graph.

Definition 1: Control flow graph of a program. The control flow graph of a program, $C F G=<N, E, n_{0}, n_{e}>$, is a directed graph $\langle N, E\rangle$ in which the nodes $N$ represent the statements and the edges $E$ indicate the conditional transfer of control between the statements. There is a path from node $n_{0} \in N$ to all other nodes, and all paths end with node $n_{e} \in N$. $n_{0}$ is called the initial node and $n_{e}$ is called the terminal node.

A path in the control flow graph traversing nodes $n_{i}$, e.g., $\left\{n_{1}, n_{2}, \cdots, n_{k}\right\}$ is called an execution trajectory [33]. The control flow graph represents the possible execution trajectories. In the control flow graph of a program, regions with a single entry and a single exit are hammock graphs. Several formal definitions for hammock graphs exist, e.g., see [14], [20]. Here, the definition from Ferrante et al. [14] is used.
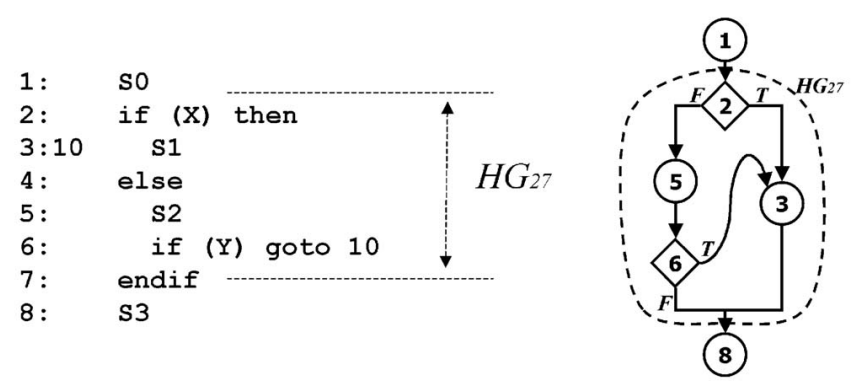

Fig. 2. A program and its control flow graph. The subgraph bounded by the dashed line is hammock graph $H G_{27}$ with entry node 2 and exit node 7 .

Definition 2: Hammock graph. Let $C F G=\left\langle N, E, n_{0}, n_{e}>\right.$ be a control flow graph and consider a subgraph $H=<N^{\prime}$, $E^{\prime}, u, v>$ with a distinguished node $u$ in $H$ and $a$ distinguished node $v$ not in $H$ such that 1) all edges from $(C F G-H)$ to $H$ go to $u, 2)$ all edges from $H$ to $(C F G-H)$ go to $v$. $H$ is called a hammock graph. Node $u$ is called the entry node and $v$ is called the exit node of $H$.

Fig. 2 shows a program, the control flow graph and a hammock graph. This example will be used to illustrate the key steps of the algorithm.

The single-entry, single-exit property of hammock graphs allow us to confine the restructuring region. Since a hammock graph transformation doesn't generate side effects on the rest of the program, the following corollary holds.

Corollary 1. A correct transformation of the control flow in a hammock graph maintains the correctness of the program.

Hereby, we remind that that a restructuring transformation does not depend on data outside the hammock graph and that code duplication and new variables as a result of the transformation are invisible for code outside the hammock graph.

Ill-structured code is identified by the type and interaction of the control statements. There are two types of control statements: block-oriented statements and branches.

Definition 3: Block-oriented and branch statements. $A$ block-oriented control statement governs the execution of a sequential block of statements delineated by language dependent markers, e.g., braces in the C-language or do, enddo in Fortran. Block-oriented statements can be nested, and the nesting level of a statement is the number of block-oriented statements guarding its execution. A branch control statement is a statement of the type: if $(<\mathrm{bcond}>)$ goto $<$ label $>$, where $<\mathrm{bcond}>$ is the branch condition and $<$ label $>$ identifies the target statement. The goto statement is called the branch, and the <label $>$ is called the target. This definition includes unconditional jumps, and statements with implicit targets, such as stop and return.

Definition 4: Branch types. A branch is classified as backward or a forward if its target occurs, respectively, before or after the branch in lexical order. The lexical order is defined as the statement ordering, also called the program ordering [2], [22]. A branch in a block-oriented statement is called incoming or outgoing when the branch and its target are not in the same block and the target is respectively inside or 


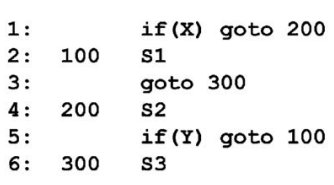

(a)

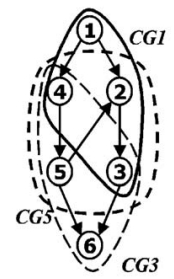

(b)

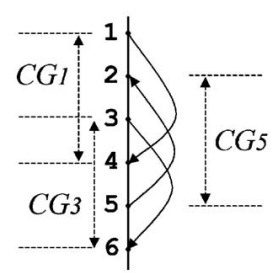

(c)
Fig. 3. An example program (a) and its control flow graph (b). The three subgraphs in (b) are the control graphs of respectively statements 1,3 , and 5. Graph (c) is a CFG where the nodes are vertically aligned according to the statement order in the program.

outside the block. For example, a branch in a while-loop with a target outside the loop body is an outgoing branch.

Definition 5: Branch order. Let $i_{f}$ be a forward branch. If no forward branch lexically occurs before $i_{f}$, then branch $i_{f}$ is called the initial forward branch. Let $i_{b}$ be a backward branch. If no backward branch lexically occurs before $i_{b}$, then branch $i_{b}$ is called the initial backward branch.

Definition 6: Structured control statement. A control statement $p$ is structured if it is block-oriented and each block of statements controlled by $p$ is a hammock graph.

Note that, according to this definition, a block-oriented statement such as a loop or an if-then-else statement is structured only if the loop body or the if-blocks are hammock graphs. For example, the block-oriented if-statement in Fig. 2 is not structured because the inner if-statement jumps from the else part to the then part. In general, whenever a block contains an incoming jump, the block becomes unstructured.

Definition 7: Control graph of a control statement. Let $C F G=<N, E, n_{0}, n_{e}>$ be a control flow graph and let node $p \in N$ represent a control statement. The control graph of $p$ is the subgraph $C G_{p}=<\Gamma, \Delta, p, q>$, where $q$ is defined as follows: 1) If $p$ is a block-oriented control statement, then $q$ is lexically the last node of the block of statements controlled by $p$; 2) if $p$ is a branch, then $q$ is the target node of $p$. Furthermore $\Gamma=\{a \mid a \in N$ and $a$ is a statement between $p$ and $q$ in the source program, including $p$ and $q\}$ and $\Delta=\{(a, b) \mid a, b \in \Gamma$ and $(a, b) \in E\}$.

Informally, the control graph $C G_{p}$ describes the region of the program directly controlled by statement $p$. Fig. 3 shows the control graphs of three branches. In Fig. 2, the control graph of the if-then-endif statement is the nodes 2-7, i.e., $H G_{27}$, while the control graph of the if-goto statement is the nodes 3-6. Control graphs may be disjoint, fully nested or they may interact.

Definition 8: Interacting control statements. Let $C G_{i_{0}}=$ $<\Gamma, \Delta, i_{0}, i_{e}>$ and $C G_{m_{0}}=<\Gamma^{\prime}, \Delta^{\prime}, m_{0}, m_{e}>$ be the control graphs of two control statements $i_{0}$ and $m_{0}$, where $i_{0} \neq m_{0}$. If the set of nodes $\Gamma$ and $\Gamma^{\prime}$ partially intersect, i.e., $\left(\Gamma \cap \Gamma^{\prime} \neq \phi\right) \wedge\left(\Gamma^{\prime} \nsubseteq \Gamma\right) \wedge\left(\Gamma \nsubseteq \Gamma^{\prime}\right)$, then control statements $i_{0}$ and $m_{0}$ interact. If $i_{0}$ interacts with $m_{0}$ and $m_{0}$ interacts with control statement $k_{0}$, then $i_{0}$ and $k_{0}$ interact indirectly.
Interacting control statements create an indeterminate control flow which at compile time prevents optimizing loop transformations because it is difficult to determine the dataflow and the dependencies. A minimal hammock graph of a branch is found by including all control graphs which interact directly or indirectly with the control graph of the branch.

Definition 9: Minimal hammock graph of a branch. Let $n_{b}$ be a branch statement in a program with control flow graph $C F G=<N, E, n_{0}, n_{e}>$. The minimal hammock graph of $n_{b}$, denoted $M H G_{n_{b}}=<N^{\prime}, E^{\prime}, m_{0}, m_{e}>$, is the smallest hammock subgraph of $C F G$, containing $n_{b}$.

The minimal hammock graph $M H G_{n_{b}}$ of a branch $n_{b}$ defines its region of control. An algorithm to find the minimal hammock graph of a branch statement $n_{b}$ is given below.

\section{Algorithm 1}

(find the minimal hammock graph of branch $n_{b}$ )

input: $n_{b} \quad$ - a branch;

output: $M H G_{n_{b}}$ - the minimal hammock graph containing $n_{b}$.

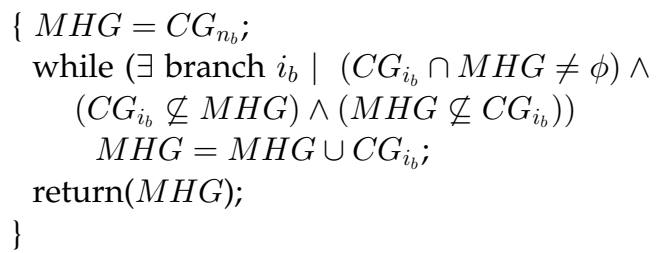

In this algorithm, the while condition selects the branches $i_{b}$ of which the control graph $C G_{i_{b}}$ partially intersects $M H G$. Applying the algorithm to the if-statement at line 5 in Fig. 3 gives iteratively statements (nodes) $\{2-5\} \cup\{1-4\} \cup\{3-6\}=\{1-6\}$.

Definition 10: Structured program and control flow graph. If all control graphs of a control flow graph are structured, i.e., are hammocks, then the contro flow graph is called complete hammock and the program is well-structured.

\section{The Conversion of Branch Statements}

The block-oriented control structures used are if-then-elseendif, while, and repeat. In this section, branches are converted into structured control statements.

A single branch. A single branch is a branch not interacting with other branches or block-oriented control statements. As such its control graph is a hammock graph. A single forward branch is replaced by a block-if and a single backward branch is converted into a while or a repeat statement. In this way, the branch is replaced by a structured control statement.

Multiple interacting branches. Interacting branches are restructured by type: first backward branches are converted into loops containing no incoming branches. In the process forward branches out of a loop are replaced by an "exit" statement followed by a jump to the target. This is repeated until no backward branches remain. Next the forward branches are converted one by one into structured if-statements. 
Branches interacting with block-oriented statements. A programing language usually contains block-oriented control statements such as do loops or if-then-else statements. When a block-oriented control statement interacts with a branch, it prevents hammock graph restructuring. As an example the if statement at line 6 in Fig. 2 interacts with the block-if statement. Therefore, first, the block-oriented if-statements are converted into branches. Then, branches out of loops are cut into exit statements inside the loop and conditional branches outside the loop. This makes the loops single-entry, single-exit hammocks. Next, the branches are removed.

\subsection{Algorithm Outline}

The hammock graph restructuring algorithm is based on three types of code transformations, respectively, for outgoing, backward, and forward branches.

The outline of the algorithm is as follows:

1. Preprocessing. Replace if-then-else statements interacting with branches by if-goto statements.

2. Single branches. Replace single branches by structured block-if or loop statements.

3. Outgoing branches. Replace an outgoing branch in a loop by an exit statement and put a conditional jump to the original target after the loop. This is the Cut transformation (Algorithm 2).

4. Backward branches. For each backward branch, determine the minimal hammock graph enclosing the branch. Starting with the initial backward branch $i_{b}$ in this graph, convert each backward branch into a loop and move the targets of incoming branches outside the loop body (Algorithm 3). Cut each outgoing forward branch in the loop into an exitjump followed by a conditional jump to the target of the branch

5. Forward branches. For each forward branch, determine the minimal hammock graph enclosing the branch. Starting with the initial forward branch, convert each forward branch into a structured if-statement (Algorithm 4).

After removal of the branches, the structuring may offer extra opportunities to remove dead code (e.g., see Fig. 7e) or to beautify the result, e.g., by using alternative looping constructs. This could be taken care of in a postprocessing step, based on specific compiler optimizations requested by the user. The following subsections present these techniques in more detail.

\subsection{Cut Transformation}

Loops with outgoing forward branches are unstructured. Therefore the Cut conversion is applied: 1) a new variable $b r_{i}$ is used to register the state of the branch condition and then the loop control expression is modified using the variable $\left.b r_{i}, 2\right)$ the long jump is cut into two parts, one is within the loop and jumps to the end of the loop using the exit statement, the other is located outside the loop and conditionally jumps to the original target. Algorithm 2 realizes this transformation.

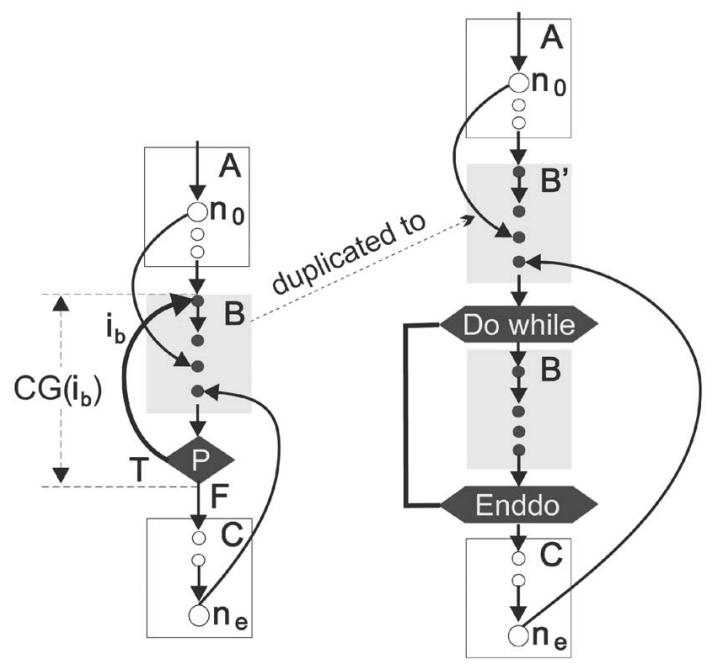

(a)

(b)

Fig. 4. Illustration of the backward branch elimination. (a) The minimal hammock graph $M H G_{i_{b}}$, initial backward branch $i_{b}$, beginning and end nodes $n_{0}$ and $n_{e}$. (b) After converting $i_{b}$ into a while statement, there are two nested hammock graphs: the body of the while loop, $B$, and the blocks $\left\{A, B^{\prime}\right.$, do while and $\left.C\right\}$.

\section{Algorithm 2 (elimination of outgoing branches)}

input: loop_nest - a loop nest with outgoing branches; output: loop_nest - a loop nest without outgoing branches.

\section{Cut(loop_nest) \{}

for $l=$ the innermost to the outermost loop

$\{n=$ the number of exit branches in loop $l$;

Before loop $l$, insert:

1. $f p_{i}=$.false. $(1 \leq i \leq n)$

For each outgoing branch $i$ with guard condition $B_{i}$ $(1 \leq i \leq n)$ :

$\left\{\right.$ replace branch $i$ to target $t_{i}$ by:

2. if $\left(B_{i}\right)$ then $\left\{f p_{i}=\right.$.true.; exit $\}$

\} After loop $l$ insert:

3. if $\left(f p_{i}\right)$ goto $t_{i}(1 \leq i \leq n)$

\}

$$
\text { \} }
$$

\subsection{Backward Copy}

Let $i_{b}$ be an arbitrary backward branch. Principally, a backward branch can be represented by a repeat-statement if there are no incoming branches into the loop body. An incoming branch prevents the backward branch from being directly converted into a loop. Since the body of this loop is executed at least once, we unroll the loop one time and modify the repeat loop into a while loop. The labels of incoming branches are moved out of the loop. As Fig. 4 illustrates, after unrolling the loop, the incoming branches $n_{0}$ and $n_{e}$ are moved outside the loop and the backward branch is converted into a while-loop. This process is called the Backward copy. However, we cannot take an arbitrary backward branch and use Backward Copy in order to remove this branch. For example, suppose in Fig. 4 we convert $n_{e}$ first instead of $i_{b}$ into a repeat-loop. Because loop overlapping is not allowed, $i_{b}$ cannot be directly converted 


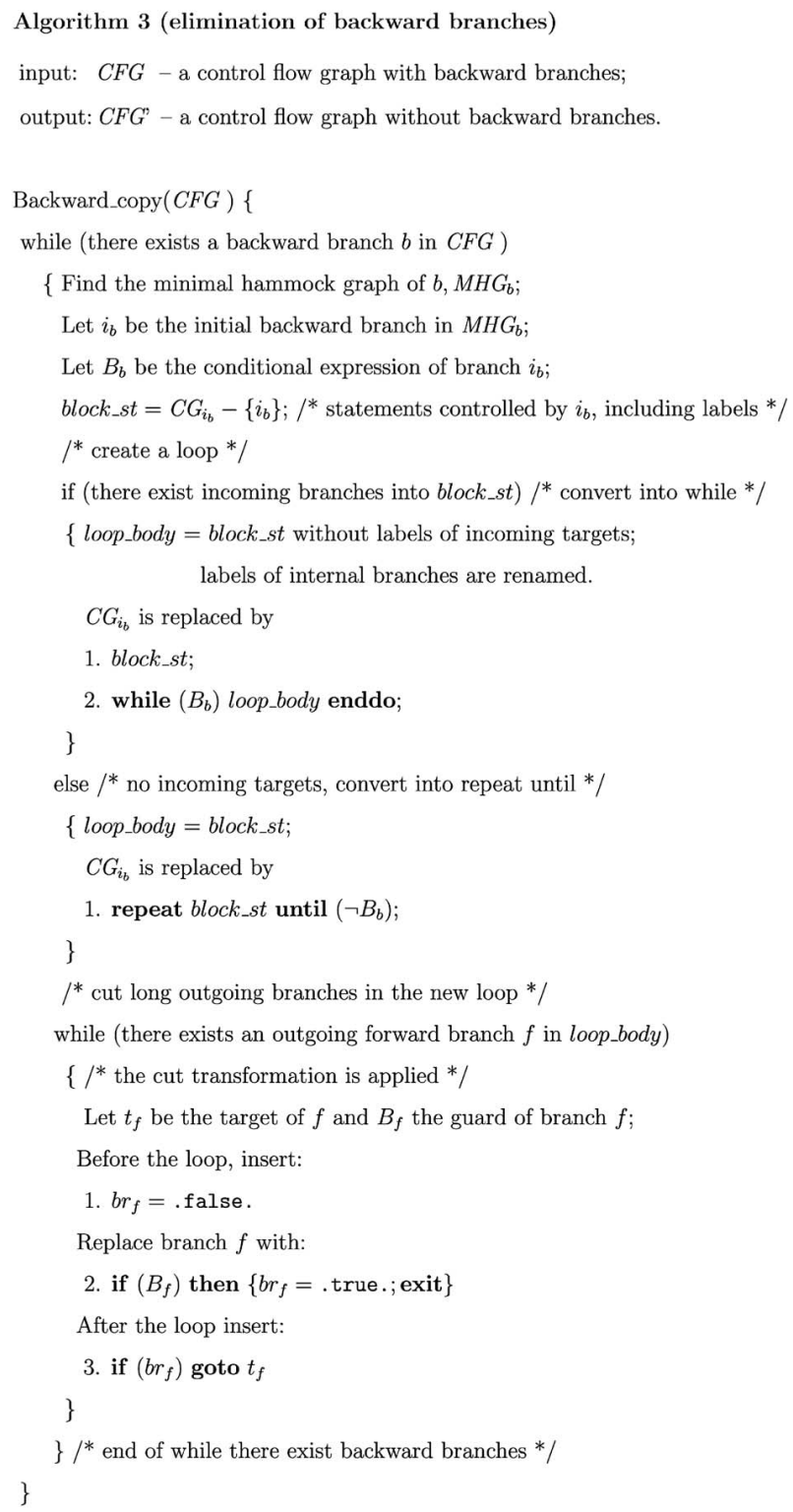

Fig. 5. Algorithm 3: Elimination of backward branches.

into either a repeat-loop or a while-loop. When the initial backward branch is converted first, there are no internal backward branches going out of the loop.

The loop_body contains only target labels of internal branches. Labels used as targets of both internal and external branches are renamed, and the internal branches are redirected to the new internal labels.

When a backward branch is converted into a loop, the only remaining outgoing branches are forward branches. These are removed using the Cut transformation. After removing the first initial backward branch, the next initial backward branch is selected and removed, and so on, until no backward branches remain. The steps are shown in Algorithm 3 (Fig. 5).

\subsection{Forward Copy}

At this point, the backward branches are converted into loops without incoming branches by Algorithm 3. Outgoing

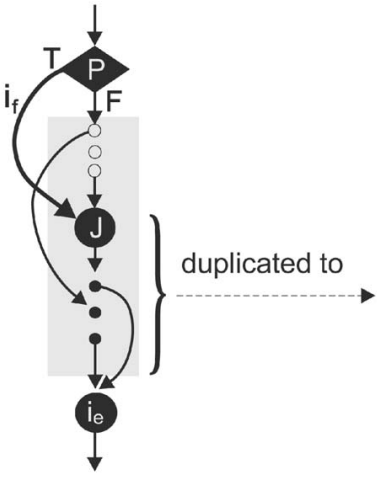

(a)

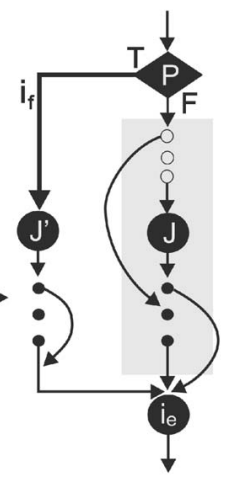

(b)
Fig. 6. (a) The minimal hammock graph $M H G_{i_{f}}$. $i_{f}$ is a forward branch whose target is $J$, and $i_{e}$ is the terminal node. (b) The shared statements are duplicated into the true part of $i_{f}$. The brace indicates the shared statements.

branches are eliminated using exit statements. As a consequence, the only remaining branches are forward branches branches both of whose end points appear at the same nesting level. A forward branch transfers control to the target or to the next statement, depending on a Boolean condition. The first trajectory is called the true_part, the other trajectory is called the false_part. Forward branches are eliminated starting with the initial forward branch. Then, for each initial branch $i_{f}$, the surrounding minimal hammock graph is determined and the initial branch is structured.

Given the initial forward branch $i_{f}$, the minimal hammock graph $M H G_{i_{f}}=<N, E, i_{f}, i_{e}>$ contains all forward branches interacting with $i_{f}$ (see Fig. 6). The statements on the path between the target of $i_{f}$ and the terminal node $i_{e}$ are called the shared statements in $M H G_{i_{f}}$. The shared statements are determined as follows: Let $C G_{i_{f}}$ be the control graph of forward branch $i_{f}$. Furthermore, let $J$ be the target of the branch $i_{f}$, then one has shared statements $=\left(M H G_{i_{f}}-C G_{i_{f}}-\left\{i_{e}\right\}\right) \cup J$. Branch $i_{f}$ is converted into a block-if where the shared statements are duplicated into the true part and the statements inside the minimal hammock graph $M H G_{i_{f}}$ are moved into the false part. The following algorithm eliminates forward branches in a program where backward branches and outgoing branches have been eliminated.

\section{Algorithm 4 (elimination of forward branches)}

input: $C F G$ - a flowgraph with only forward branches; output: $C F G^{\prime}$ - a flowgraph without branches.

\section{Forward_copy $(C F G)\{$}

$\left\{\right.$ while (there exists an initial forward branch $i_{f}$ )

$\left\{\right.$ Find the minimal hammock graph of $i_{f}, M H G_{i_{f}}$, with end node $i_{e}$;

if $\left(C G_{i_{f}}\right.$ interacts with other forward branches)

$\left\{I^{*}\right.$ a forward-copy transformation is applied * true_part $=$ the shared statements $=$

$$
\left(M H G_{i_{f}}-C G_{i_{f}}-\left\{i_{e}\right\}\right) \cup J \text {; }
$$$$
\text { false_part }=M H G_{i_{f}}-\left\{i_{f}, i_{e}\right\} \text {; }
$$

$M H G_{i_{f}}$ is replaced by: 


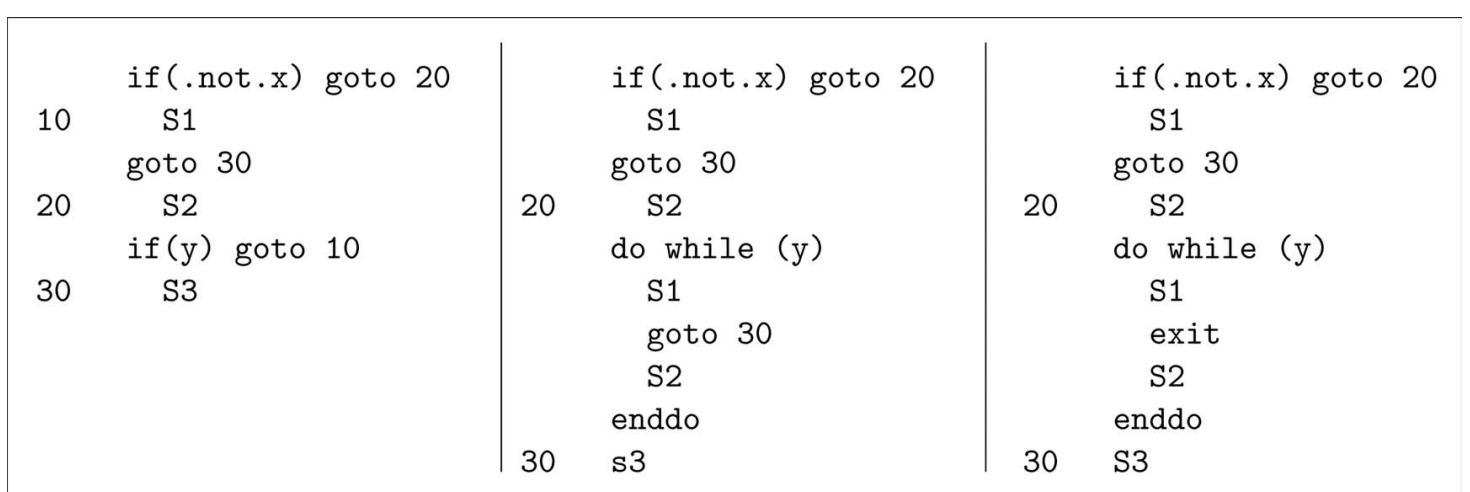

(a)

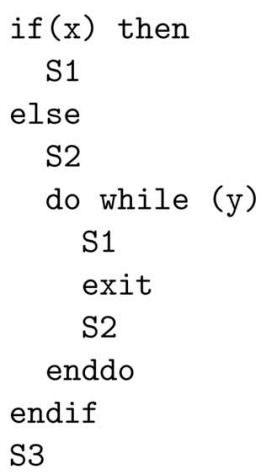

(d) (b)

if $(x)$ then
S1
else
S2
if(y) then
$\quad$ S1
endif
endif
S3

(c)

Fig. 7. Restructuring steps of the unstructured code inside the hammock graph $H G_{27}$ in Fig. 2. (a) Conversion into branches, (b) backward copy, (c) cut outgoing branch, (d) forward copy, (e) unconditional exit optimization.

1. if $(B)$ then true_part else false_part endif;

\}$/{ }^{*} B$ is the Boolean expression of $i_{f} .^{*} /$

else ${ }^{*}$ a single forward branch */

$\left\{\right.$ true_part $=M H G_{i_{f}}-\left\{i_{f}, i_{e}\right\}$;

$M H G_{i_{f}}$ is replaced by:

1. if $(\neg B)$ then true_part endif; $\left\{i_{e}\right\}$; \}

Eliminate_forward(true_part);

Eliminate_forward(false_part);

Example. The algorithm is applied to the hammock graph $H_{27}$ of Fig. 2. Fig. 7 shows the successive steps:

1. First, the block-if is converted into branches because the then-part contains an incoming branch.

2. Next, the backward copy creates a while loop without incoming branch.

3. The cut transformation converts the outgoing branch into an exit. Since the branch and while loop have the same successor, no logical variable is necessary.

4. The forward copy removes the last branch. The shared statements in the then-part are eliminated due to dead code removal.

5. Finally, the compiler replaces the single whileloop iteration by a block-if statement.
In the following section, the correctness of the basic hammock graph transformations is formally proven using axiomatic proof rules.

\section{Correctness Proof of the Elementary Program Transformations}

The elementary transformations used to construct hammock graphs are limited to the Forward copy, the Backward copy, and the Cut transformation. These transformations operate on a branch by branch basis and each handles a particular type of interacting branch. Since no other transformations are used, the basic cases cover all branches. In this section, the correctness proofs of these transformations are developed based on an axiomatic program specification [18], [17], [24], [1]. The formulas of the inference system have the form $\{P\} S\{Q\}$, where $S$ is a block of statements representing a hammock graph. Branches are either within $S$ or are expressed explicitly. $P$ is a precondition and $Q$ is a postcondition. The proof rules for structured statements are given in Table 1. Unstructured statements containing a goto to label $L$ have the form:

$$
\{P\} \text { if }(B) \text { goto } L\{Q\}\{L: R\} .
$$

There are two postconditions: $\{Q\}=\{P \wedge \neg B\}$ for the normal exit and $\{L: R\}=\{L: P \wedge B\}$ for the jump to the statement labeled $L:$. If the computation state satisfies 
TABLE 1

Axiomatic Proof Rules for Structured Control Statements

\begin{tabular}{|c|c|}
\hline $\begin{array}{l}\text { consequence } \\
\text { rules }\end{array}$ & $\begin{array}{l}\{P\} S\{R\}, R \Rightarrow Q \\
\{P\} S\{Q\} \\
\{P\} \Rightarrow R,\{R\} S\{Q\} \\
\{P\} S\{Q\}\end{array}$ \\
\hline $\begin{array}{l}\text { compound } \\
\text { rule }\end{array}$ & $\frac{\left\{P_{i-1}\right\} S_{i}\left\{P_{i}\right\}, \text { for } i=1 \ldots n}{\left\{P_{0}\right\} S_{1} ; S_{2} ; \ldots ; S_{n}\left\{P_{n}\right\}}$ \\
\hline if rule & $\begin{array}{c}\{P \wedge B\} S_{1}\{Q\},\{P \wedge \neg B\} S_{2}\{Q\} \\
\{P\} \text { if }(B) \text { then } S_{1} \text { else } S_{2} \text { endif }\{Q\} \\
\{P \wedge B\} S\{Q\},\{P \wedge \neg B\} \Rightarrow\{Q\} \\
\{P\} \text { if }(B) \text { then } S \text { endif }\{Q\}\end{array}$ \\
\hline while rule & $\frac{\{P \wedge B\} S\{P\}}{\{P\} \text { do while }(B) S \text { enddo }\{P \wedge \neg B\}}$ \\
\hline $\begin{array}{l}\text { repeat } \\
\text { rule }\end{array}$ & $\begin{array}{c}\{P\} S\{Q\},\{Q \wedge \neg B\} \Rightarrow\{P\} \\
\{P\} \text { repeat } S \text { until }(B)\{Q \wedge B\}\end{array}$ \\
\hline
\end{tabular}

precondition $P$, then on a normal exit, $Q$ is asserted, while on the jump goto $L$, the computation state satisfies $R$. In addition, $R$ is an invariant condition which is satisfied for all computing states reaching label $L$. The inference rules for goto's are given in Table 2 .

The case goto $L$ is treated as if (true) goto $L$. Applying the goto rule, this gives: $\{P\}$ goto $L\{$ false $\}\{L: P\}$.

Definition 11: Semantic equivalence. Let $\{P\}$ be an arbitrary precondition. $S$ and $S^{\prime}$ are programs, and $\{P\} S\{Q\}$, $\{P\} S^{\prime}\left\{Q^{\prime}\right\}$. The program $S$ is semantically equivalent to the program $S^{\prime}$, if and only if $Q=Q^{\prime}$.

Definition 12: Invariant condition. An invariant condition $U$ is a predicate at label $L$ in the program such that all incoming computation states satisfy $U$. If an incoming computation state $s$ at $L$ satisfies predicate $R$, then one has $R \Rightarrow U$.

Theorem 1: Backward copy. The Backward copy transformation preserves the semantics of the program.

Proof. A Backward copy is applied when there exists a target for an incoming branch within the control region of an initial backward branch, such as label $L$ in the program shown in Fig. 8a. Denote $L$ the target of an incoming branch. The Backward copy converts the program (a) (Fig. 8) into (b) (Fig. 8). Suppose $\{P\}$ (Fig. 8a) $\{Q\}$ and $\{P\}$ (Fig. 8b) $\left\{Q^{\prime}\right\}$. Let $U$ and $R_{2}$ be the invariant conditions respectively before $S_{1}$ and after $S_{2}$ in program (a) (Fig. 8). Now, location $L$ has two or more incoming states: the state after $S_{1}$ and the state of an

TABLE 2

Inference Rules for Labels and Goto's

\begin{tabular}{||l|l||}
\hline \hline goto rule & $\{P\}$ if $(B)$ goto $L\{P \wedge \neg B\}\{L: P \wedge B\}$ \\
\hline compound (1) & $\frac{\{P\} S_{1}\left\{Q_{1}\right\}\{L: R\},\left\{Q_{1}\right\} S_{2}\left\{Q_{2}\right\}\{L: R\}}{\{P\} S_{1} ; S_{2}\left\{Q_{2}\right\}\{L: R\}}$ \\
\hline compound (2) & $\frac{\{P\} S_{1}\left\{Q_{1}\right\}\left\{L_{1}: R_{1}\right\},\left\{Q_{1}\right\} S_{2}\left\{Q_{2}\right\}\left\{L_{2}: R_{2}\right\}}{\{P\} S_{1} ; S_{2}\left\{Q_{2}\right\}\left\{L_{1}: R_{1}\right\}\left\{L_{2}: R_{2}\right\}}$ \\
\hline backward labeling & $\frac{\{P\} S_{1}\{R\}\{L: R\}}{\{P\} S_{1} L:\{R\}}$ \\
\hline forward labeling & $\frac{\{R\} S_{1}\{Q\}\{L: R\}}{\{R\} L: S_{1}\{Q\}}$ \\
\hline \hline
\end{tabular}

\begin{tabular}{clc}
\hline$L_{1}:$ & $S_{1} ;$ & \\
$L:$ & $S_{2} ;$ & $S_{1}$ \\
& if $(B)$ goto $L_{1}$ & $S_{2}$ \\
& & do while $(B)$ \\
& & $S_{1}$ \\
& & $S_{2}$ \\
& enddo \\
& (a) & (b)
\end{tabular}

Fig. 8. Elementry backward copy transformation: (a) backward branch, (b) do while conversion.

arbitrary incoming branch to target $L$. Let $R_{1}$ be a condition which is satisfied by those states. Hence, $\{U\} S_{1}\left\{R_{1}\right\},\left\{R_{1}\right\} L: S_{2}\left\{R_{2}\right\}$. Furthermore, $U$ is an invariant condition at the label $L_{1}$ and all incoming states must satisfy $U$, therefore $R_{2} \wedge B \Rightarrow U$ and $P \Rightarrow U$. The proof rules of the program (a) (Fig. 8) are given in Fig. 9. With $P \Rightarrow U$ and $R_{2} \wedge B \Rightarrow U$, the consequence and the forward labeling rules give: $\{P\} \quad L_{1}: S_{1} L: S_{2}$ if $(B)$ goto $L_{1} \quad\left\{R_{2} \wedge \neg B\right\}$. Hence, $\{Q\}=\left\{R_{2} \wedge \neg B\right\}$. Using $U$ and $R_{i}$ as defined in Fig. 9, rewriting the clauses and applying the axiomatic proof rules, program (b) (Fig. 8) is derived as shown in Fig. 10: Since the postcondition of program (b) (Fig. 8b), $Q^{\prime}=R_{2} \wedge \neg B=Q$, the transformed program is semantically equivalent to the original. Furthermore, the incoming target is moved out of the loop.

Theorem 2 (Cut transformation). The Cut transformation preserves the semantics of the program.

Proof. The Cut transformation breaks a branch out of a loop into two branches. The first branch just exits the loop, the second is located after the loop and transfers the control to the target of the original far jump. In this way, the program (a) (Fig. 11) is transformed into (b) (Fig. 11). To prove that the transformed program (b) (Fig. 11) is semantically equivalent to the program (a) (Fig. 11), let $\{P\}$ (Fig. 11a) $\{Q\}$ and $\{P\}$ (Fig. 11b) $\left\{Q^{\prime}\right\}$. Assume $U$ is an invariant condition before the loop. Furthermore, define $R_{i}, i=1, \ldots, 3$, such that $\left\{U \wedge B_{1}\right\} S_{1}\left\{R_{1}\right\},\left\{R_{1} \wedge\right.$ $\left.\neg B_{2}\right\} S_{2}\{U\}$ and $\left\{U \wedge \neg B_{1}\right\} S_{3}\left\{R_{3}\right\}$. Because $U$ is an invariant condition before the loop, one has $\{P\} \Rightarrow\{U\}$. Now, the program (a) (Fig. 11) is annotated with the assertions shown in Fig. 12. Hence, the postcondition at $L_{2}$ is $Q=R_{3} \vee R_{1} \wedge B_{2}$.

In program (b) (Fig. 11), the instruction exit is introduced. Semantically, this instruction modifies the postcondition of the dowhile construct, $\left\{U \wedge \neg B_{1}\right\}$, by adding the disjunction of the exit condition, $R_{e}$, giving rise to the new postcondition, $\left\{U \wedge \neg B_{1} \vee R_{e}\right\}$. A new logical variable $b r_{2}$ is introduced to register the state of the Boolean expression $B_{2} . b r_{2}$ is outside the state space of the original program. Therefore, neither $S_{1}$ nor $S_{2}$ nor $S_{3}$ change the state of $b r_{2}$, i.e.,

$\left\{R_{i-1}\right\} S_{k}\left\{R_{i}\right\} \Rightarrow\left\{R_{i-1} \wedge b r_{2}\right\} S_{k}\left\{R_{i} \wedge b r_{2}\right\},(1 \leq k \leq 3)$. 


\begin{tabular}{lll}
$P \Rightarrow U,\{U\}$ & $L_{1}: S_{1}$ & $\left\{R_{1}\right\}$ \\
$\left\{R_{1}\right\}$ & $L: S_{2}$ & $\left\{R_{2}\right\}$ \\
$\left\{R_{2}\right\}$ & if $(B)$ goto $L_{1}$ & $\left\{R_{2} \wedge \neg B\right\}\left\{L_{1}: R_{2} \wedge B \Rightarrow U\right\}$ \\
\hline$P \Rightarrow U,\{U\}$ & $L_{1}: S_{1} L: S_{2}$ if $(B)$ goto $L_{1}$ & $\left\{R_{2} \wedge \neg B\right\}$ \\
& & $\left\{L_{1}: U\right\} \quad$ (compound rule)
\end{tabular}

Fig. 9. Computing the postcondition of Fig. 8a.

Using the proof rules of program (a) (Fig. 11), program (b) (Fig. 11) can be derived next (see Fig. 13). $L_{w}$ is a placeholder for the exit-node of the while-loop.

The postcondition of program (b) (Fig. 11) is $Q^{\prime}=R_{3} \wedge \neg b r_{2} \vee R_{1} \wedge B_{2} \wedge b r_{2}$. Since the branch variable $b r_{2}$ can take either value true. or .false., depending on the program, one has $Q^{\prime} \Rightarrow R_{3} \vee R_{1} \wedge B_{2}$. Therefore, $Q^{\prime} \Rightarrow Q$ and the transformation is valid.

Since a repeat statement can be represented by a while statement, it can be proven similarly that the Cut transformation in the case of a repeat statement is valid.

Theorem 3: (Forward copy). The Forward copy transformation preserves the semantics of the program.

Proof. Consider program (a) (Fig. 14) containing interacting forward branches. The forward branches have targets $L_{1}$ and $L_{2}$, respectively. The minimal hammock graph of the initial forward branch extends from the first statement to target label $L_{2}$. The Forward copy converts the initial forward branch into a block-if statement by duplicating the shared statement $S_{3}$ into the true part of the block-if (see Fig. 6). The resulting program is given in (Fig. 14b). The second noninteracting forward branch is similarly converted into a block-if, resulting program (c) (Fig. 14). Let $\{P\}$ (Fig. 14a) $\{Q\}$ and $\{P\}$ (Fig. 14c) $\left\{Q^{\prime}\right\}$. Now, define $R_{i}$ in program (a) (Fig. 14) as follows: Suppose $\left\{P \wedge \neg B_{1}\right\} S_{1}\left\{R_{1}\right\}$, denote $R_{2}$ and $R_{3}$ the invariant preconditions at the label $L_{1}$ and $L_{2}$ and let $\left\{R_{3}\right\} S_{4}\left\{R_{4}\right\}$. Then, one has $\left\{R_{1} \wedge \neg B_{2}\right\} S_{2}\left\{R_{2}\right\}, P \wedge B_{1}$ $\Rightarrow R_{2},\left\{R_{2}\right\} S_{3}\left\{R_{3}\right\}$, and $R_{1} \wedge B_{2} \Rightarrow R_{3}$.

The proof rules of (Fig. 14a) are shown in Fig. 15. Hence, the postcondition of program (a) (Fig. 14a) is $Q=R_{4}$. From these proof rules, the transformed program (c) (Fig. 14c) is derived as shown in Fig. 16. By applying the if-rule twice, the labels are removed and the postcondition of the transformed program becomes

\begin{tabular}{lll}
\hline$P \Rightarrow U,\{U\}$ & $S_{1}$ & $\left\{R_{1}\right\}$ (Fig. 8a) \\
$\left\{R_{1}\right\}$ & $L: S_{2}$ & $\left\{R_{2}\right\}$ (Fig. 8a) \\
$\left\{R_{2} \wedge B\right\}$ & $S_{1}$ & $\left\{R_{1}\right\}$ (consequence) \\
$\left\{R_{1}\right\}$ & $S_{2}$ & $\left\{R_{2}\right\}$ (Fig. 8a) \\
\hline$\{P\}$ & $S_{1} L: S_{2}$ & \\
& do while $(B)$ & \\
& $S_{1} S_{2}$ enddo & $\left\{R_{2} \wedge \neg B\right\}$ \\
\hline
\end{tabular}

Fig. 10. Computing the postcondition of Fig. 8b.
$Q^{\prime}=R_{4}=Q$. Consequently, the forward copy transformation is correct.

\section{Results}

The algorithms have been implemented in the research parallelizing compiler FPT [12], [37] with the aim to structure programs and to extract the parallelism in illstructured programs. Four file sets were selected, respectively, from the single-precision Linpack suite, the singleprecision Lapack suite, the double-precision Eispack suite, and the single-precision Slap benchmark. Table 3 lists a statistical analysis of the benchmarks, showing the relative code expansion (excluding comments and format directives), the new control variables and the parallel loops. The average code expansion is 32 percent, which is comparable to Erosa's 22 percent code expansion obtained for a different benchmark [13]. The Linpack suite has a very modest code increase. A closer look reveals that every branch target is a continue statement, which is removed after restructuring. In addition, most goto's are used to implement if-then-else-endif constructs. This requires an extra

\begin{tabular}{|c|c|}
\hline do while $\left(B_{1}\right)$ & $\begin{array}{l}b r_{2}=\text { false. } \\
\text { do while }\left(B_{1}\right)\end{array}$ \\
\hline$S_{1}$ & $S_{1}$ \\
\hline if $\left(B_{2}\right)$ goto $L_{2}$ & if $\left(B_{2}\right)\left\{b r_{2}=\right.$. true.; exit $\}$ \\
\hline$S_{2}$ & $S_{2}$ \\
\hline enddo & enddo \\
\hline$S_{3}$ & if $\left(b r_{2}\right)$ goto $L_{2}$ \\
\hline$L_{2}:$ & $S_{3}$ \\
\hline & \\
\hline (a) & (b) \\
\hline
\end{tabular}

Fig. 11. Elementry cut transformation: (a) loop with outgoing branch, (b) conversion using the exit statement.

\begin{tabular}{lll}
$\{P \Rightarrow U\}$ & dowhile $\left(B_{1}\right)$ & $\left\{U \wedge B_{1}\right\}$ \\
$\left\{U \wedge B_{1}\right\}$ & $S_{1}$ & $\left\{R_{1}\right\}$ \\
$\left\{R_{1}\right\}$ & if $\left(B_{2}\right)$ goto $L_{2}$ & $\left\{R_{1} \wedge \neg B_{2}\right\}\left\{L_{2}: R_{1} \wedge B_{2}\right\}$ \\
$\left\{R_{1} \wedge \neg B_{2}\right\}$ & $S_{2}$ & $\{U\}$ \\
& enddo & $\left\{U \wedge \neg B_{1}\right\}$ \\
$\left\{U \wedge \neg B_{1}\right\}$ & $S_{3}$ & $\left\{R_{3}\right\}$ \\
$\left\{R_{3} \vee R_{1} \wedge B_{2}\right\}$ & $L_{2}:$ & \\
\hline
\end{tabular}

Fig. 12. Computing the postcondition of Fig. 11a. 


\begin{tabular}{|c|c|c|c|}
\hline$\{P\}$ & & $b r_{2}=$ false & $\left\{P \wedge \neg b r_{2}\right\}$ \\
\hline$\left\{P \wedge \neg b r_{2} \Rightarrow U \wedge \neg b r_{2}\right\}$ & & dowhile $\left(B_{1}\right)$ & $\left\{U \wedge B_{1} \wedge \neg b r_{2}\right\}$ \\
\hline$\left\{U \wedge B_{1} \wedge \neg b r_{2}\right\}$ & & $S_{1}$ & $\left\{R_{1} \wedge \neg b r_{2}\right\}$ \\
\hline$\left\{R_{1} \wedge \neg b r_{2}\right\}$ & & if $\left(B_{2}\right)$ then & $\left\{R_{1} \wedge B_{2} \wedge \neg b r_{2}\right\}$ \\
\hline \multirow[t]{2}{*}{$R_{1} \wedge B_{2} \wedge \neg b r_{2}$} & & $b r_{2}=. t r u e . ;$ exit & $\left\{L_{w}: R_{1} \wedge B_{2} \wedge b r_{2}\right\}$ \\
\hline & & endif & $\left\{R_{1} \wedge \neg B_{2} \wedge \neg b r_{2}\right\}$ \\
\hline \multirow[t]{2}{*}{$R_{1} \wedge \neg B_{2} \wedge \neg b r_{2}$} & & $S_{2}$ & $\left\{U \wedge \neg b r_{2}\right\}$ \\
\hline & & enddo & $\left\{U \wedge \neg B_{1} \wedge \neg b r_{2}\right\}$ \\
\hline$\left\{U \wedge \neg B_{1} \wedge \neg b r_{2} \vee R_{1} \wedge B_{2} \wedge b r_{2}\right\}$ & $L_{w}:$ & if $\left(b r_{2}\right)$ goto $L_{2}$ & $\left\{U \wedge \neg B_{1} \wedge \neg b r_{2}\right\}$ \\
\hline$\left\{U \wedge \neg B_{1} \wedge \neg b r_{2}\right\}$ & & $S_{3}$ & $\left\{R_{3} \wedge \neg b r_{2}\right\}$ \\
\hline$\left\{R_{3} \wedge \neg b r_{2} \vee R_{1} \wedge B_{2} \wedge b r_{2}\right\}$ & $L_{2}:$ & & \\
\hline
\end{tabular}

Fig. 13. Computing the postcondition of Fig. 11b.

jump from then then-part to the end of the if-block, which is also removed after restructuring. Since the Linpack routines contain only reducible loops and are quite structured, there is almost no code expansion. More than half of the routines has a slight code reduction, while, in 80 percent of the routines, the code expansion is limited to eight lines. A few programs contain large "computed goto's" with interfering targets, causing a lot of code duplication. The largest increase of 48 percent is for the routine ss.for. This routine contains a computed-goto (multijump) statement with 19 targets, pointing to overlapping code blocks. On the other hand, the code expansion is highest for the Eispack benchmark, with an average of 85.3 percent. This is generated by a handful routines with highly interacting branches and many target labels creating a maximal code increase of 5.5, while the median of the code increase is eight lines. Many Lapack routines have no goto's and were left out of the test suite. The Lapack benchmark routines used here have an average code size of 192 lines and generate a limited code increase of 26.2 percent, with a median of seven lines. The Slap benchmark has an average code size of 303 lines, with a median expansion of 11 lines.

After removing the branches, while- and repeat-loops are generated. For parallelization, these loops need to be converted into DO-loops. This conversion requires two

\begin{tabular}{|cccc}
\hline \multicolumn{1}{|c}{ if $\left(B_{1}\right)$ goto $L_{1}$} & if $\left(B_{1}\right)$ then & if $\left(B_{1}\right)$ then \\
& $S_{1}$ & $S_{3}$ & $S_{3}$ \\
if $\left(B_{2}\right)$ goto $L_{2}$ & else & else \\
& $S_{2}$ & $S_{1}$ & $S_{1}$ \\
$L_{1}: S_{3}$ & if $\left(B_{2}\right)$ goto $L_{2}$ & if $\left(\neg B_{2}\right)$ then \\
$L_{2}: S_{4}$ & $S_{2}$ & $S_{2}$ \\
& & $S_{3}$ & $S_{3}$ \\
& & & endif \\
& & endif & endif \\
& & $L_{4}$ & $S_{4}$ \\
& (a) & (b) & (c)
\end{tabular}

Fig. 14. Elementry forward copy transformation: (a) interacting forward branches, (b) applying the forward copy eliminates the initial forward branch, (c) simple conversion of the remaining branch. steps: 1) the definition of an induction variable which serves as index of the loop and 2) the determination of the loop count. A technique for the conversion of while into do-loops was developed, which is described in [38]. The FPT compiler does not parallellize programs containing goto's because of the potential side-effects prohibiting the dependency analysis. After removing the goto's, normally a substantial number of parallel loops is detected. In order to estimate the impact of branch removal on the detection of additional parallelism, the parallel loops are classified into two groups. Parallelizable loops not interacting with branches are classified as "parallel loops before restructuring." Loops parallelized after branch removal are called "parallel loops after restructuring." The Table 3 shows that the present restructuring technique allows between 10 percent and 30 percent extra loops to be parallelized in the Linpack, Eispack, and Lapack routines. Slap is already better structured and, therefore, the gain is only 2 percent.

Code size increase can reduce performance, e.g., loop unrolling is known to increase cache misses and register pressure which may offset the benefit of the transformation. This is especially important when the amount of memory available is limited, e.g., in embedded systems. However, in most cases, the code size increase is limited and the parallelism outweighs the code size penalty. In extreme cases, rather than reverting to unstructured code, new alternatives at the link level offer efficient and uniform code compaction techniques [10].

The structuring of ill-structured programs is recognized as a necessary condition for many compiler optimizations, especially for the exploitation of instruction level parallelism [19], vectorization [2], predicated instruction scheduling [8], contemporary VLIW architectures [32], and the

\begin{tabular}{|c|c|c|c|}
\hline $\begin{array}{l}\{P\} \\
\left\{P \wedge \neg B_{1}\right\}\end{array}$ & & $\begin{array}{l}\text { if }\left(B_{1}\right) \text { goto } L_{1} \\
S_{1}\end{array}$ & $\begin{array}{l}\left\{P \wedge \neg B_{1}\right\}\left\{L_{1}: P \wedge B_{1} \Rightarrow R_{2}\right\} \\
\left\{R_{1}\right\}\end{array}$ \\
\hline$\left\{R_{1}\right\}$ & & if $\left(B_{2}\right)$ goto $L_{2}$ & $\left\{R_{1} \wedge \neg B_{2}\right\}\left\{L_{2}: R_{1} \wedge B_{2} \Rightarrow R_{3}\right\}$ \\
\hline$\left\{R_{1} \wedge \neg B_{2}\right\}$ & & $S_{2}$ & $\left\{R_{2}\right\}$ \\
\hline$\left\{R_{2}\right\}$ & $L_{1}:$ & $S_{3}$ & $\left\{R_{3}\right\}$ \\
\hline$\left\{R_{3}\right\}$ & $L_{2}:$ & $S_{4}$ & $\left\{R_{4}\right\}$ \\
\hline
\end{tabular}

Fig. 15. Computing the postcondition of Fig. 14a. 


\begin{tabular}{|c|c|c|c|}
\hline$P \wedge B_{1} \Rightarrow R_{2},\left\{R_{2}\right\}$ & & $S_{3}$ & $\left\{R_{3}\right\}$ (Fig. 14a) \\
\hline$\left\{P \wedge \neg B_{1}\right\}$ & & $S_{1}$ & $\left\{R_{1}\right\}$ (Fig. 14a) \\
\hline$\left\{R_{1}\right\}$ & & if $\left(B_{2}\right)$ goto $L_{2}$ & $\left\{R_{1} \wedge \neg B_{2}\right\}\left\{L_{2}: R_{1} \wedge B_{2} \Rightarrow R_{3}\right\}$ (Fig. 14a) \\
\hline$\left\{R_{1} \wedge \neg B_{2}\right\}$ & & $S_{2}$ & $\left\{R_{2}\right\}$ (Fig. 14a) \\
\hline$\left\{R_{2}\right\}$ & & $S_{3}$ & $\left\{R_{3}\right\}$ (Fig. 14a) \\
\hline$\left\{R_{3}\right\}$ & $L_{2}:$ & $S_{4}$ & $\left\{R_{4}\right\}$ (Fig. 14a) \\
\hline \multirow[t]{8}{*}{$P$} & & if $\left(B_{1}\right)$ then & (apply the if-rule) \\
\hline & & $S_{3}$ & \\
\hline & & else & \\
\hline & & $S_{1}$ & \\
\hline & & if $\left(\neg B_{2}\right)$ then & (apply the if-rule) \\
\hline & & $S_{2} S_{3}$ & $\left\{R_{3}\right\}$ \\
\hline & & endif & \\
\hline & & endif & \\
\hline$\left\{R_{3}\right\}$ & $L_{2}:$ & $S_{4}$ & $\left\{R_{4}\right\}$ \\
\hline
\end{tabular}

Fig. 16. Computing the postcondition of Fig. 14c.

handling of control flow in general. In this respect, the results of our hammock graph restructuring technique has a number of characteristics which compare favorably with other approaches. This comparison is elaborated in the section about related work.

\section{Related Work}

Structuring programs has been an active research topic for many years, with shifting methods and objectives, see Table 4. In the earlier papers by Boehm et al. [7], Peterson et al. [29], Ashcroft and Manna [5], and Williams [34], [36], a number of techniques were introduced based on flowcharts. Boehm et al. [7] define a set of functional and predicative boxes, similar to sequential and control statements and show that any flowchart (a two-dimensional programming language) can be normalized by three basic constructs, composition, selection and iteration, in such a way that all loops are properly nested. The results are applied to the theory of Turing machines, but no algorithm for ordinary

TABLE 3

Restructuring Four Benchmark Suites

\begin{tabular}{||l|c|c|c|c|c||}
\hline \hline & Linpack & Lapack & Eispack & Slap & Total \\
\hline Lines B & 7379 & 24546 & 4557 & 3039 & 39521 \\
Lines A & 8255 & 30980 & 8444 & 4481 & 52160 \\
\hline Lines Incr. & $11.9 \%$ & $26.2 \%$ & $85.3 \%$ & $47.4 \%$ & $32.0 \%$ \\
\hline \hline Gotos B & 714 & 563 & 602 & 71 & 1950 \\
\hline \hline Par. loops B & 322 & 476 & 323 & 91 & 1212 \\
Par. loops A & 355 & 561 & 419 & 93 & 1428 \\
\hline Par. loops Incr. & 33 & 85 & 96 & 2 & 216 \\
\hline Par. loops Incr. \% & $10.2 \%$ & $17.9 \%$ & $29.7 \%$ & $2.2 \%$ & $17.8 \%$ \\
\hline \hline New vars & 7 & 161 & 150 & 32 & 350 \\
\hline \hline Files & 60 & 128 & 67 & 10 & 265 \\
\hline \hline
\end{tabular}

$B=$ before, $A=$ after, Incr. = increment, Par. = parallel loops, Par. loops Incr. = unstructured loops parallelized after restructuring, New vars = new logical variables. programs is given. Ashcroft and Manna [5] introduce two algorithms to remove goto's in a flowchart using while statements. The first algorithm (not described in the paper) duplicates code, the second algorithm tries to find cut-sets within the flow graph, to eliminate the duplication using extra logical variables. The hammock graph approach uses selectively repeat and while loops, leading to no code replication and no extra logical variables in the example flow charts of [3]. Peterson et al. [29] have shown that for any given flowchart, a program can be written using if-statements, repeat statements, and multilevel exit statements. The chart may have to be modified by node splitting. A "well-formed program" is defined as one in which loops and conditional statements are properly nested and entered only at their beginning. Starting from a flowchart, an algorithm is given which transforms a flowchart into a wellformed flowchart. A second algorithm produces a wellformed program from a well-formed flowchart. Besides starting from a flowchart, the difference with our approach is that multilevel exits are actually goto's jumping to the

\section{TABLE 4}

Chronological List of Restructuring Methods

\begin{tabular}{||l|l|l|}
\hline Input & Approach & IMP \\
\hline Turing machine [7] & composition, iteration & \\
Flowchart [5] & while statements & \\
Flow diagram [36] & repeat, multi-level exits, RP & \\
Program [2] & ifstructured base diagrams & \\
Schema [26] & if-conversion & PFC \\
Program [30] & multiple exit statements, RP & \\
Program [3] & factoring continuations & MIPRAC \\
Program [13] & goto relocation and elimination & Mc-CAT \\
Binary [9] & interval analysis & dcc \\
Program (this paper) & nested hammock graphs & FPT \\
\hline
\end{tabular}

$C D G=$ control dependence graph, $R P=$ reducible program only, $I M P=$ compiler implementation. 
start of an arbitrary enclosed loop. This may confuse the reader or complicate the dependence analysis, and is indeed considered a cause of unstructuredness in [36]. Furthermore, very few languages support the multilevel exit construct. Williams and Ossher [36] state that a structured flow diagram consists of three base diagrams: the simple sequence, the selection (if-then-else), and the while loop. Unstructuredness is identified by five flow graph structures, one structure of interacting forward branches called abnormal selection path, and four loops structures, denoted respectively, multiple entry, multiple exit, overlapping, and parallel loops. A detailed algorithm is given to find the loop and selection structures and substitute the anomalous structures by structured ones. This mechanical restructuring doesn't always produce the most efficient or most pleasing solution. In particular, the bare flowchart of a program looses information about the structured loops and if-statements available in the source. In a later paper [35], goto's are removed from a Pascal program, taking advantage of the limitations of the use of goto's in this language and considering three basic sets of transformations. Besides being related to a particular programming language, the examples of the paper use more temporary variables (flags) than in our approach. Furthermore, no benchmark results such as code expansion are given. The if-conversion technique developed by Allen et al. [2] is aimed at vectorizing Fortran loops. The idea is to remove control statements and replace them by guarded Fortran $8 \mathrm{X}$ vector instructions, e.g., WHERE $(\operatorname{BR} 1(1: N)) A(1: N)=B(1: N)+$ 10. A number of transformations on branches are applied. Branch relocation moves branches out of loops until the branch and its target are at the same loop nesting level; in this way, they become a forward or backward branch. Branch removal eliminates forward branches by computing guard expressions for statements under their control and conditioning execution on these expressions. Backward branches are left in place because they imply loops which cannot be expressed by guarded statements. If-conversion has two limitations: First, it is only oriented towards vectorization, and second, backward branches representing loops with potential parallelism are not converted. In our approach, we follow a similar classification of branches into forward, backward, and exit branches; however, the control restructuring is done at the hammock graph level, instead of the guarded statement level. This allows a coarse grain parallelization as well as vectorization. In addition, backward branches are converted into loops amenable to parallelization. Ramshaw [30] wants to eliminate goto's while maintaining the program structure. He proves that removing goto's without code duplication requires a reducible program. Based on a stricter set of ground rules than Peterson et al. [29], goto's are eliminated for a conforming program. However, the stricter rules imply that a major cause of unstructured loops, i.e., irreducible loops with multiple entries, is unaccounted for.

A notably different approach is the use of continuations as presented by Ammarguellat [3]. Informally, a continuation is the remaining execution trajectory at any particular point in the program. Ammarguellat uses continuations as unknowns at the branch points in the program. This results in a set of continuation equations which can be solved recursively in a Gaussian elimination style. When the solution is found, code factorization allows to replace multiple identical continuation codes executable under different execution conditions by a single continuation code, executed under a generalized condition. This factoring allows to avoid all code replication, except for the cases of irreducible graphs. The approach by Ammarguellat is quite elegant and feasible, but rather complex compared to the approach presented here. Moreover, by factoring the continuation code, the execution conditions become complex and the associated overhead might become noticeable, especially in small inner loops. In contrast, our technique may create a relatively small code replication, but the branch expressions remain essentially the same as in the original program. Furthermore, the goal to avoid code replication generates complex predicate expressions because the same code, executed under different conditions, will appear only once in the program. While this indeed creates a more compact code, the execution performance can be hampered by a lengthy predicate analysis. Consider the following example from Ammarguellat (Fig. 17a). The Lisp program is rewritten into Fortran, in order to use our FPT compiler, and the original condition (i.gt.0) is replaced by (i.gt.5) , to avoid dead code between labels 20 and 40. Ammarguellat's normalized flow program (Fig. 17b) contains a double repeat loop, three predicates, and seven conditional expressions representing in total 12 logical operations. In contrast, the hammock graph transformation generates two loops, two predicates, four conditional expressions, and three logical operations in total. In addition, instead of the outer repeat loop in Fig. 17b, a do while loop without predicates in the loop condition is generated (see Fig. 17c). The simple loop condition, derived from the if-statement, allows the loop to be converted into a parallel do-loop Fig. 17d, using induction variable detection and dependence analysis [38]. This example illustrates that reducing and simplifying branch conditions enhances the granularity and may allow more optimization, an observation already made by Allen et al. [2].

Oulsnam [25] has an interesting paper in which he uses Kleene's algebra to describe an unstructured flow graph and reduce it to a structured form represented by structured regular expressions. He extended Brainbridge's reduction rules, by introducing logical variables to remember the values of branch conditions in the unstructured flowgraph. In this way, the flowgraph can be structured by applying the reduction rules to a system of so-called "endset" equations. No algorithm is given, but there is a followup paper on the algorithmic transformation of schemes [26] and the regular expression paradigm was recently revisited [23]. The problem with this approach is that a lot of extra logical variables are introduced, which unnecessarily increase the loop nesting level and lower the granularity of the resulting program. As an example, consider Oulsnam's treatment of the generalized Flynn's problem No. 5 (Fig. 7 in [25]). For the comparison, the flowchart is 


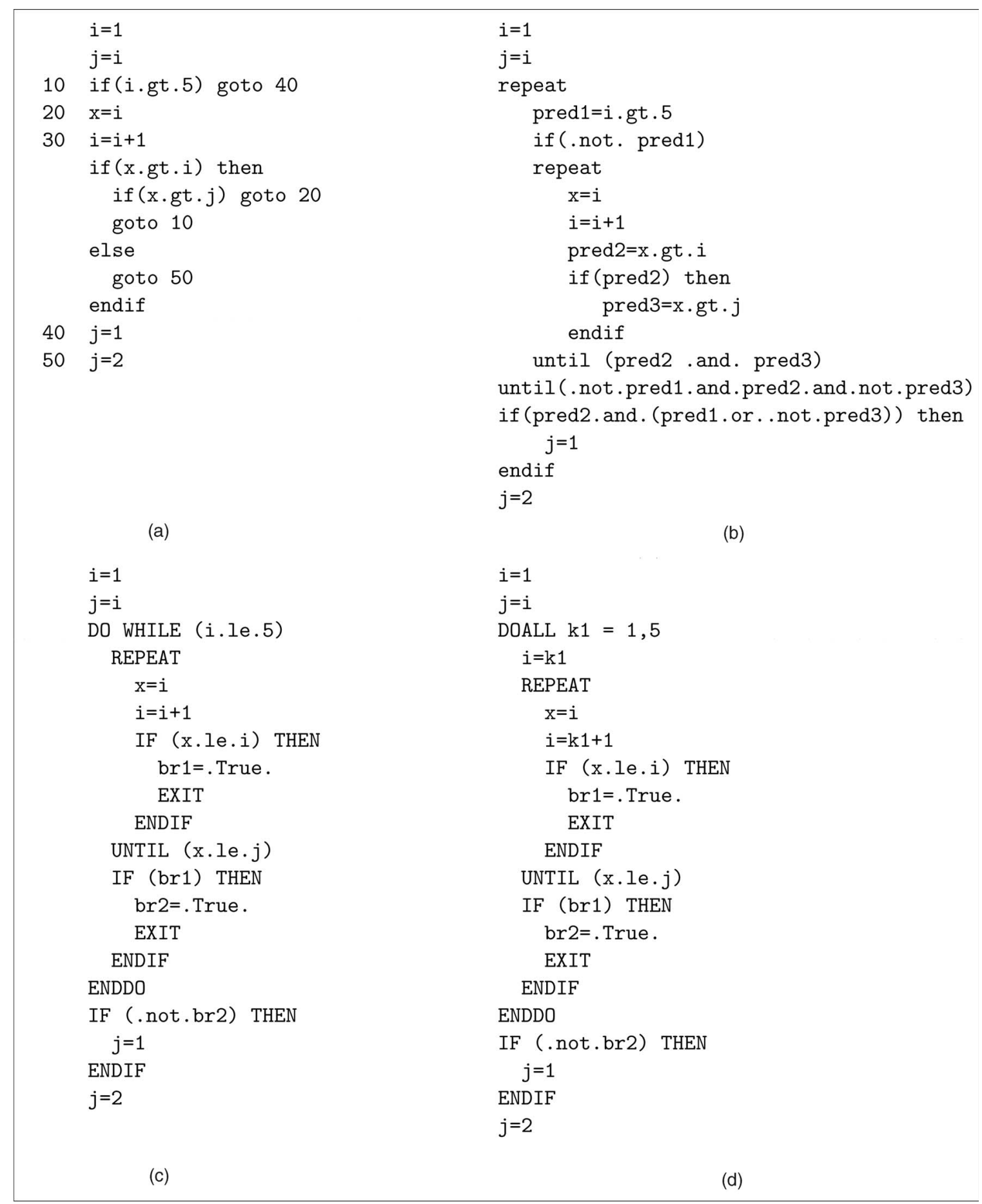

Fig. 17. (a) Program with three branches, (b) minimizing code replication using predicates [3], (c) limited code replication using hammock graph restructuring reduces predicate proliferation, and (d) allows induction variable detection and parallel loop conversion.

rephrased in a Fortran style, with case sensitive variable names. From left to right one has the original program, the code derived from Oulsnam's flowchart and the code generated by our transformer, FPT, see Fig. 18. The unstructured program contains 16 lines; the programs restructured by regular expression reduction and by hammock graph transformations, respectively, contain 33 and 43 lines. Although Oulsnam's code is 24 percent shorter, it is not necessarily more efficient. This is because the control structure is more deeply nested and requires more conditions to be checked before executing code. For example, the maximum nesting depth of the different assignment statements is given in Table 5 .

A large nesting depth means more jumps and less efficient pipelining, especially in loops. Moreover, the basic blocks are smaller, e.g., the average size of an execution block is 1.18 statements in Oulsnam's version and 1.85 statements in the hammock graph transformation. A large granularity is necessary to extract parallelism for wide issue architectures [8]. 


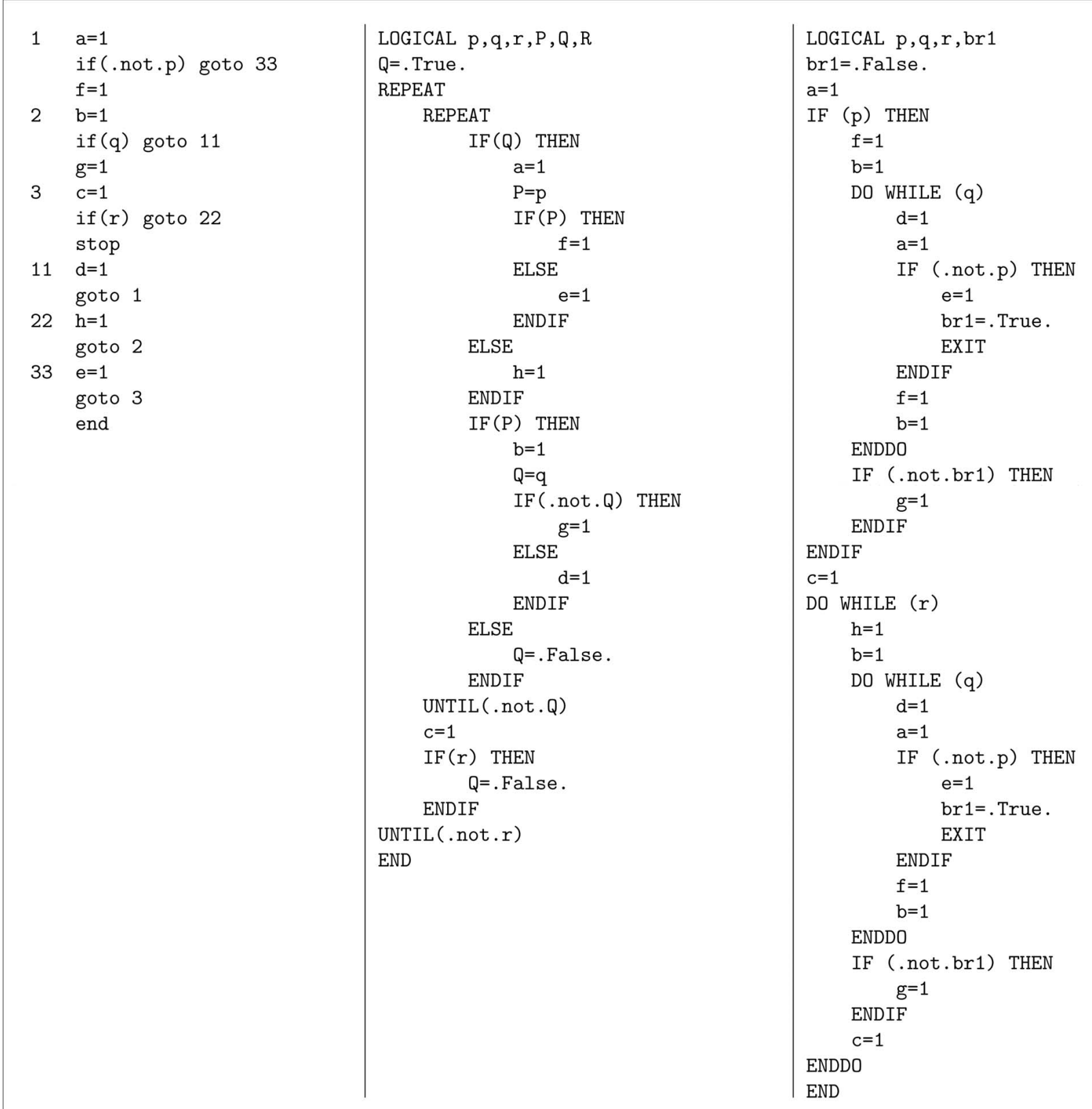

(a)

(b)

(c)

Fig. 18. (a) example from Oulsnam [25], (b) code restructuring using Kleene's algebra, and (c) Hammock graph restructuring.

TABLE 5

Maximum Nesting Depth with Two Restructuring Approaches

\begin{tabular}{l|ccccccc|c}
\hline Max. nesting depth & $\mathrm{a}=1$ & $\mathrm{~b}=1$ & $\mathrm{c}=1$ & $\mathrm{~d}=1$ & $\mathrm{e}=1$ & $\mathrm{f}=1$ & $\mathrm{~g}=1$ & average \\
\hline Regular expressions [25] & 3 & 3 & 1 & 4 & 4 & 4 & 4 & 3.28 \\
Hammock graphs & 2 & 2 & 1 & 2 & 3 & 2 & 2 & 2.00 \\
\hline
\end{tabular}

With respect to implementation, Allen and Ammarguellat have implemented the branch removal into a Fortran compiler, whereas Eroasa and Hendren [13] describe an implementation for a C-compiler. Erosa and Hendren follow basically the same branch classification as Allen, but offer a different implementation for the exit-jumps: They are moved in or out of the loop or if-block such that branch and target are at the same nesting level. Incoming jumps are cut into hops, and a conditional jump is placed at the start of each block. Whereas the treatment of exit jumps is the same as our approach, we treat the problem of irreducibility differently: Loops and blocks with incoming jumps are immediately converted into a set of interacting branches. This allows the backward and the forward 


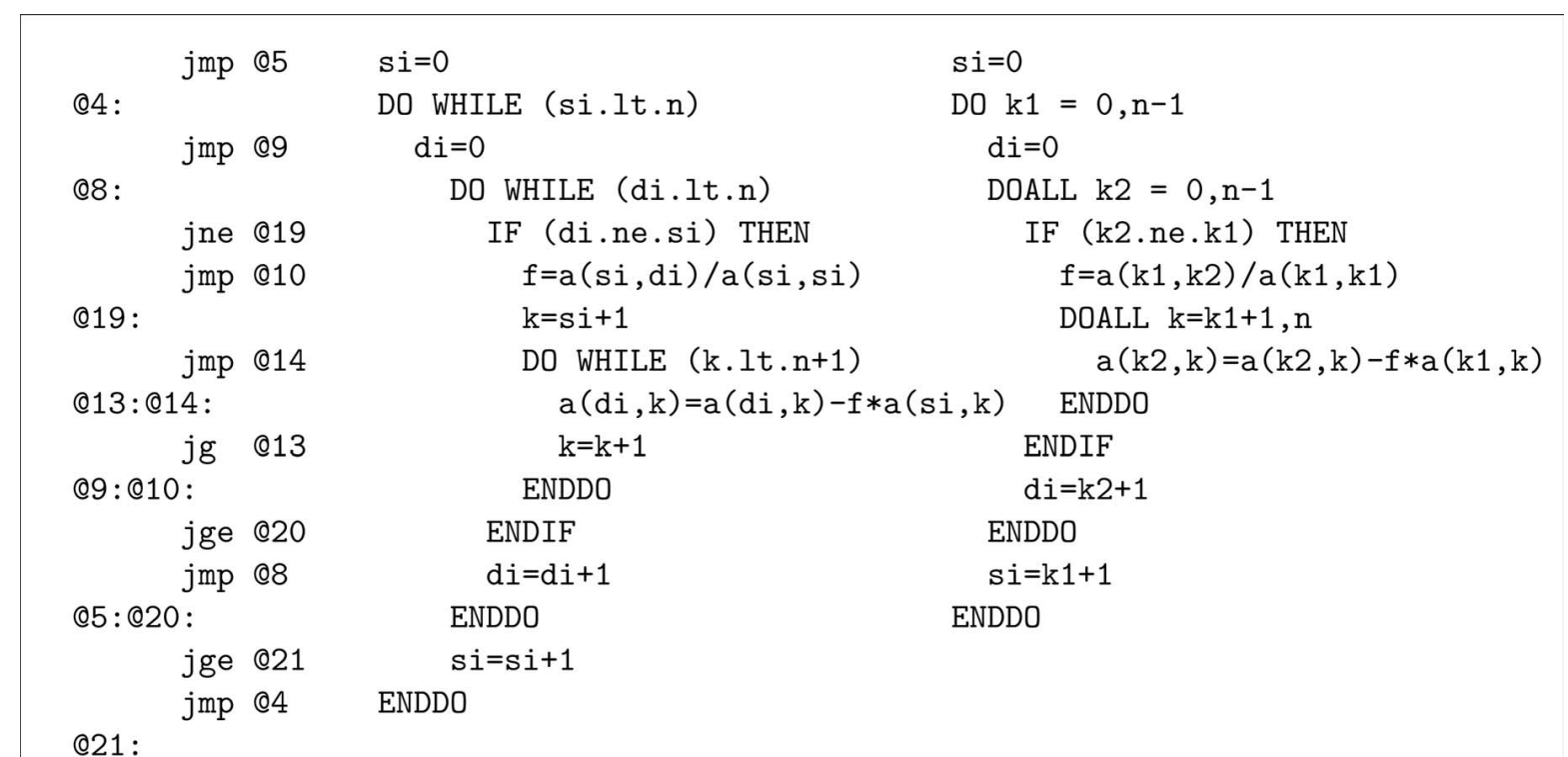

(a)

(b)

(c)

Fig. 19. Decompiling and parallelizing an assembler program. (a) Branches and labels from the assembler listing of the Gauss-Jordan elimination. (b) After restructuring with this paper's algorithm, the backward branches become while loops. (c) By removing the induction variables two while loops are converted into parallel loops.

transformations to eliminate the irreducibility without the need for extra control variables.

A recent application area is structuring decompiled binary programs. Cifuentes [9] developed the decompiler dcc, able to convert 8086 executable code into a C program, with a limited number of goto's. To illustrate the use of hammock graph restructuring in this area, we take the assembly output from the Gauss-Jordan linear system solver, generated by Turbo-C. The elimination loop contains 10 branches and 15 labels, which are depicted in Fig. 19a (other code is omitted). After rewriting the program in Fortran, taking into account the array dimensions and applying the hammock graph restructuring with FPT, the program in Fig. 19b is obtained in which the goto's are removed and the backward jumps become while loops. In order to parallelize the nested loop, two further steps are needed: the detection of an induction variable and a data dependence analysis. Using the technique described in [37], [38], FPT finds two parallel DOALL-loops, see Fig. 19c.

Except for the algebraic treatment of continuations by Ammarguellat, none of the methods to remove branches have been formally proven. This is quite understandable since a formal proof for all but simple programs has been recognized to be quite difficult [17]. On the other hand, the quality of the compiler, as ubiquitous translation layer to steer the machine hardware, is of utmost importance. Fortunately, the painstaking effort to devise a rigorous proof can now be alleviated with the advent of increasingly capable automatic theorem provers. PVS, the prototype verification system of SRI [28], has been successfully used to verify the correctness of program transformations [31], mathematical theorems [6] as well as live-critical aviation software [27]. In our approach of program restructuring, the actual program transformation is carried out by three basic transformations. We were able to prove that the transformations are correct and the correctness proofs were verified using PVS [11]. The proof uses Arbib et al.'s extension of the axiomatic rules for goto's [4].

\section{Conclusion}

Structured programs are necessary for readability and for effective compiler optimizations. In this paper, simple, efficient and provable correct basic program transformations are presented to remove all branches in a program. A restructuring algorithm using the three elementary transformations converts the control flow graph of a program into a nest of hammock graphs.

From the comparison with related work it becomes apparent that there is a trade off between conditional expression complexity, code granularity and code replication. The transformations presented allow a limited code replication in order to keep the sequential blocks intact and to minimize the pressure on predicate resources and branch prediction logic. The effect has been measured on several benchmarks, using an implementation of the hammock graph transformations in the research parallelizing compiler FPT [37].

\section{RefERENCES}

[1] S. Alagic and M.A. Arbib, The Design of Well-Structured and Correct Programs. Springer, 1978.

[2] J.-R. Allen, K. Kennedy, C. Porterfield, and J. Warren, “Conversion of Control Dependence to Data Dependence," Proc. 10th Ann. ACM Symp. Principles of Programming Languages, pp. 177-189, Jan. 1983.

[3] Z. Ammarguellat, "A Control-Flow Normalization Algorithm and Its Complexity," IEEE Trans. Software Eng., vol. 18, no. 3, pp. 237251, 1992. 
[4] M.A. Arbib and S. Alagic, "Proof Rules for Gotos," Acta Informatica, vol. 11, pp. 139-148, 1979.

[5] E. Ashcroft and Z. Manna, "The Translation of Goto Programs into While Programs," Proc. IFIP Congress 71, C. Freiman, J. Griffith, and J. Rosenfeld, eds., vol. 1, pp. 250-255, 1972.

[6] B. Dutertre, "Elements of Mathematical Analysis in PVS," Proc. Ninth Int'l Conf. Theorem Proving in Higher Order Logics TPHOL, J. Von Wright, J. Grundy, and J. Harrison, eds., Aug. 1996.

[7] C. Boehm and G. Jacopini, "Flow Diagrams, Turing Machines and Languages with Only Two Formation Rules," Comm. ACM, vol. 9, no. 5, pp. 366-371, May 1966.

[8] L. Carter, B. Simon, B. Calder, L. Carter, and J. Ferrante, "Path Analysis and Renaming for Predicated Instruction Scheduling," Int'l J. Parallel Programming, vol. 28, no. 6, pp. 563-588, 2000.

[9] C. Cifuentes, "Structuring Decompiled Graphs," Lecture Notes in Computer Science, vol. 1060, pp. 91-104, 1996.

[10] B. De Bus, D. Kästner, D. Chanet, L. Van Put, and B. De Sutter, "Post-Pass Compaction Techniques," Comm. ACM, vol. 46, no. 8, pp. 41-46, 2003.

[11] E.H. D'Hollander and F. Zhang, "Restructuring Program Transformations with Proof Verification Using PVS," Technical Report RUG/ELIS01, p. 47, 2001.

[12] E.H. D'Hollander, F. Zhang, and Q. Wang, “The Fortran Parallel Transformer and Its Programming Environment," Information Sciences, vol. 106, nos. 3-4, pp. 293-317, 1998.

[13] A.M. Erosa and L.J. Hendren, "Taming Control Flow: A Structured Approach to Eliminating Goto Statements," Proc. Fifth Int'l Conf. Computer Languages, pp. 229-240, 1994.

[14] J. Ferrante, K.J. Ottenstein, and J.D. Warren, "The Program Dependence Graph and Its Use in Optimization," ACM Trans. Programming Languages and Systems, vol. 9, no. 3, pp. 319-349, 1987.

[15] P. Havlak, "Nesting of Reducible and Irreducible Loops," ACM Trans. Programming Languages and Systems (TOPLAS), vol. 19, no. 4, pp. 557-567, 1997.

[16] M.S. Hecht and J.D. Ullman, "Characterizations of Reducible Flow Graphs," J. ACM, vol. 21, no. 3, pp. 367-375, 1974.

[17] C.A.R. Hoare, "An Axiomatic Basis of Computer Programming," Comm. ACM, vol. 12, pp. 576-580, 1969.

[18] C.A.R. Hoare, "An Axiomatic Definition of the Programming Language Pascal," Acta Informatca, vol. 2, pp. 335-355, 1973.

[19] J. Janssen and H. Corporaal, "Making Graphs Reducible with Controlled Node Splitting," ACM Trans. Programming Languages and Systems, vol. 19, no. 6, pp. 1031-1052, 1997.

[20] V.N. Kas'janov, "Distinguishing Hammocks in a Directed Graph," Soviet Math. Doklady, vol. 16, no. 5, pp. 448-450, 1975.

[21] A. Klauser, T. Austin, D. Grunwald, and B. Calder, "Dynamic Hammock Predication for Non-Predicated Instruction Set Architectures," Proc. 1998 Int'l Conf. Parallel Architectures and Compilation Techniques (PACT '98), pp. 278-285, Oct. 1998.

[22] L. Lamport, "How to Make a Multiprocessor Computer that Correctly Executes Multiprocess Programs," IEEE Trans. Computers, vol. 28, no. 9, pp. 690-691, 1979.

[23] P. Morris, R.A. Gray, and R.E. Filman, "GOTO Removal Based on Regular Expressions," J. Software Maintenance-Research and Practice, vol. 9, no. 1, pp. 47-66, Jan./Feb. 1997.

[24] H.R. Nielson and F. Nielson, Semantics with Applications. John Wiley and Sons, 1993.

[25] G. Oulsnam, "Unravelling Unstructured Programs," The Computer J., vol. 25, no. 3, pp. 379-387, Aug. 1982.

[26] G. Oulsnam, "The Algorithmic Transformation of Schemas to Structured Form," The Computer J., vol. 30, no. 1, pp. 43-51, Feb. 1987.

[27] S. Owre, J. Rushby, N. Shankar, and F. von Henke, "Formal Verification for Fault-Tolerant Architectures: Prolegomena to the Design of PVS," IEEE Trans. Software Eng., vol. 21, no. 2, pp. 107125, Feb. 1995.

[28] S. Owre, J.M. Rushby, and N. Shankar, "PVS: A Prototype Verification System," Proc. 11th Int'l Conf. Automated Deduction (CADE-11), D. Kapur, ed., June 1992.

[29] W.W. Peterson, T. Kasami, and N. Tokura, "On the Capabilities of While, Repeat, and Exit Statements," Comm. ACM, vol. 16, no. 8, pp. 503-512, Aug. 1973.

[30] L. Ramshaw, "Eliminating Goto's While Preserving Program Structure," J. ACM, vol. 35, no. 4, pp. 893-920, Oct. 1988.

[31] N. Shankar, "Steps Towards Mechanizing Program Transformations Using PVS," Science of Computer Programming, vol. 26, nos. 13, pp. 33-57, May 1996.
[32] S. Unger and F. Mueller, "Handling Irreducible Loops: Optimized Node Splitting Versus DJ-Graphs," ACM Trans. Programming Languages and Systems (TOPLAS), vol. 24, no. 4, pp. 299-333, 2002.

[33] M. Weiser, "Program Slicing," IEEE Trans. Software Eng., vol. 10, no. 4, pp. 352-357, July 1984.

[34] M.H. Williams, "Generating Structured Flow Diagrams: The Nature of Unstructuredness," The Computer J., vol. 20, no. 1, pp. 45-50, Feb. 1977.

[35] M.H. Williams and G. Chen, "Restructuring Pascal Programs Containing Goto Statements," The Computer J., vol. 28, no. 2, pp. 134-137, 1985.

[36] M.H. Williams and H.L. Ossher, "Conversion of Unstructured Flow Diagrams to Structured Form," The Computer J., vol. 21, no. 2, pp. 161-167, 1978.

[37] F. Zhang, "FPT: A Parallel Programming Environment," PhD thesis, Dept. of Electrical Eng., Univ. of Ghent, 1996.

[38] F. Zhang and E.H. D'Hollander, "Extracting the Parallelism in Programs with Unstructured Control Statements," Proc. Int'l Conf. Parallel and Distributed Systems, (ICPADS '94), pp. 264-270, Dec. 1994.

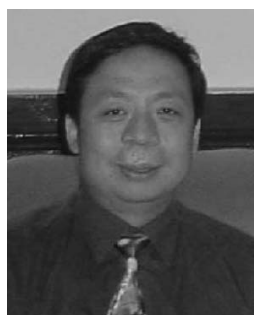

Fubo Zhang graduated from Fudan University of Shanghai (1986). He received the PhD degree from the University of Ghent in 1996. He studied in the Electronic Engineering Department of Gent University, Belgium, majoring in parallel processing and distributed computer systems (1991-1996). After graduating from the University of Ghent, he worked at Platform Computing Canada as a product architect and development team manager (1996-2001). In 2001, he relocated to Beijing, China, and worked in the Platform Beijing office as a senior professional service and support manager for A/P. His current focus is on technical computing grid technology and distributed computing resource management.

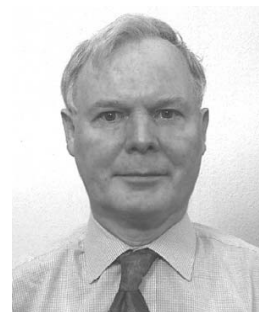

Erik H. D'Hollander graduated from the universities of Ghent, (electrical engineering) in 1972 and Leuven (computer science) in 1976. He did research on parallel processing at the Computer Science Department of the University of California at Los Angeles (1979-1980) and received the $\mathrm{PhD}$ degree from the University of Ghent, Belgium in 1980. In the summers of 1983 and 1985, he was visiting researcher, respectively, at the Department of Computer Science of UCLA and at the Center of Supercomputing Research and Development (CSRD) of the University of Illinois at Urbana-Champaign. His research interests include automatic parallelization of ordinary programs, performance computing and compiler techniques for explicitly parallel computing, multimedia and embedded system architectures. He is a member of the IEEE.

$\triangleright$ For more information on this or any other computing topic, please visit our Digital Library at www.computer.org/publications/dlib. 